\title{
Can the Arrow of Time be understood from Quantum Cosmology?
}

\author{
Claus Kiefer \\ Institut für Theoretische Physik, Universität zu Köln, Zülpicher Straße 77, 50937 \\ Köln, Germany. kiefer@thp.uni-koeln.de
}

Summary. I address the question whether the origin of the observed arrow of time can be derived from quantum cosmology. After a general discussion of entropy in cosmology and some numerical estimates, I give a brief introduction into quantum geometrodynamics and argue that this may provide a sufficient framework for studying this question. I then show that a natural boundary condition of low initial entropy can be imposed on the universal wave function. The arrow of time is then correlated with the size of the Universe and emerges from an increasing amount of decoherence due to entanglement with unobserved degrees of freedom. Remarks are also made concerning the arrow of time in multiverse pictures and scenarios motivated by dark energy.

To appear in The Arrow of Time, edited by L. Mersini-Houghton and R. Vaas (Springer, Berlin, 2010).

\section{Introduction}

The fundamental laws of physics, as they are presently known, are mostly invariant with respect to a reversal of time: to every solution there exists an equally viable solution in which $t$ is replaced by $-t$. The only exceptions are some processes described by the weak interaction, but these cases can also be subsumed under time-reversal invariance in the broad sense because its violation there can directly be compensated by an application of a unitary CP-transformation.

Despite this fundamental invariance, most classes of phenomena observed in Nature distinguish a specific direction of time. These are the famous "arrows of time" which are discussed at length in [1], see also [2] and other contributions to this volume. This observed discrepancy between the symmetric laws and the asymmetric facts does not constitute an inconsistency; asymmetric phenomena are compatible with symmetric laws, and most solutions of the 
fundamental equations are not symmetric by themselves. What is peculiar is the fact that the time direction of the phenomena is always the same.

Usually one distinguishes between various manifestations of the arrow of time 1. The electrodynamic arrow expresses the preference of the retarded over the advanced solutions. The thermodynamic arrow is given by the nondecrease of entropy for closed systems, as expressed by the Second Law of thermodynamics. The electrodynamic arrow can, in fact, be derived from the Second Law by using the thermodynamical properties of absorbers. The quantummechanical arrow expresses a direction through the measurement process or, in the Everett picture, the branching of the wave function. A central role is there played by decoherence - the irreversible and unavoidable emergence of classical properties through interaction with the environment. Finally, gravitational systems exhibit a preferred direction either through gravitational collapse or through the expansion of the Universe. The question raised by the presence of all these arrows is whether a common master arrow of time is behind all of them.

One might wonder whether the arrow of time points into different directions for different subsystems of the Universe, for example, for different galaxy clusters. Using arguments that can be traced back to Emile Borel in the 1920s, one can recognize that there is no strict isolation of subsystems and that therefore all arrows in the Universe must point into the same direction. This suggests that the master arrow of time may be found in cosmology.

Can one explain the presence of these distinguished time directions in the framework of physics? Is there a master arrow of time and, if yes, where does it come from? One might speculate that a new, hitherto unknown, fundamental law exists, which is asymmetric in time. Models of wave-function collapse are explicit examples for such new laws. However, no empirical evidence exists for them. The alternative to such a speculation is the presence of a distinguished cosmic boundary condition of low entropy at or near the Big Bang. One would then expect that the entropy of the Universe increases in the direction of increasing cosmic size. The question remains, however, where such a boundary condition could come from.

As indicated by the singularity theorems of general relativity, a consistent description of the Big Bang may require a new framework such as quantum gravity. The question then arises whether the origin of the arrow of time can be understood there. This is the topic of my essay. I shall start in the next section by making more precise the arguments in favour of a cosmic boundary condition of low entropy. I shall then present a framework of quantum gravity in which the above question can be addressed - quantum geometrodynamics, the direct quantization of Einstein's theory of general relativity. In the last section I shall then present how, in fact, the origin of irreversibility could be understood from quantum cosmology. I shall also speculate there about possible quantum effects and the fate of the Second Law in the future of our Universe. The Appendix contains numerical estimates concerning the entropy and the maximal entropy of our Universe. 


\section{Entropy and Cosmology}

Already Ludwig Boltzmann had speculated that the Second Law has its origin in a gigantic fluctuation in the Universe. His picture was that the Universe is eternally existing and at its maximal entropy most of the time, but that at very rare occasions (which, of course, can happen in an eternal Universe) the entropy fluctuates to a very low value from which it will then increase; this would then enable our existence and lead to the arrow of time that we observe. The weak point in this argument was disclosed in the 1930s by Carl Friedrich von Weizsäcker: if one takes into account the possibility of entropy fluctuations, a fluctuation that produces at once the world that we observe including our existence and our memories, although by itself extremely unlikely, would still be much more probable than Boltzmann's fluctuation which has to create the whole history of the world in addition to the present state. Strange observers which according to such a fluctuation could spontaneously pop into existence have recently been discussed in the context of a "multiverse" picture and there been called "Boltzmann brains", cf. [3] and the references therein. The multiverse picture can be motivated by inflationary scenarios of the early Universe (e.g. Linde's "eternal inflation") and describes the full Universe as being infinitely extended and very inhomogeneous on large scales, but containing many Friedmann subuniverses of the kind that we observe. In such a gigantic Universe, even the tiniest entropic fluctuation would occur somewhere. I shall briefly address the multiverse picture below, but focus in the following on the observable part of the Universe, which is approximately homogeneous and isotropic.

In order to know how special our Universe in fact is, one would like to calculate both the actual entropy of our Universe as well as the maximal possible entropy. The non-gravitational entropy is dominated by the photons of the Cosmic Microwave Background (CMB) radiation; it contributes about $2 \times 10^{89} k_{\mathrm{B}}$ 4. Linde and Vanchurin have, moreover, given an estimate of an upper limit for the non-gravitational entropy, which would be obtained if all particles were ultra-relativistic: their value is about $10^{90} k_{\mathrm{B}}$ and thus only about one order of magnitude more than the CMB value [5]. These are very large numbers, but they are much smaller than the gravitational contribution to the entropy. Unfortunately, a general expression for gravitational entropy does not exist. Because of the universal attractivity of gravity, one can only expect that gravitational entropy increases during a gravitational collapse, in contrast to the entropic trend of ordinary matter which prefers a homogeneous state. However, for the most extreme case of gravitational collapse, an entropy formula exists: the Bekenstein-Hawking entropy for black holes. It reads

$$
S_{\mathrm{BH}}=\frac{k_{\mathrm{B}} c^{3} A}{4 G \hbar}=k_{\mathrm{B}} \frac{A}{4 l_{\mathrm{P}}^{2}},
$$


where $A$ is the surface area of the event horizon and $l_{\mathrm{P}}=\sqrt{G \hbar / c^{3}}$ is the Planck length; in the following estimates we shall set Boltzmann's constant $k_{\mathrm{B}}$ equal to one.

To see how large the Bekenstein-Hawking entropy can become, let us estimate its value for the Galactic Black Hole - the supermassive black hole in the centre of our Milky Way with a mass $M \approx 3.9 \times 10^{6} M_{\odot}$. Neclecting its angular momentum, which would anyway decrease the estimated entropy, one gets from (1)

$$
S_{\mathrm{GBH}}=\pi\left(\frac{R_{\mathrm{S}}}{l_{\mathrm{P}}}\right)^{2} \approx 6.7 \times 10^{90},
$$

where $R_{\mathrm{S}}$ denotes the Schwarzschild radius. This already exceeds by more than one order of magnitude the non-gravitational contribution to the entropy. According to a recent investigation [4, all supermassive black holes together yield an entropy of $S=3.1_{-1.7}^{+3.0} \times 10^{104}$.

Roger Penrose has pointed out in [6] that the maximal entropy for the observable Universe would be obtained if all its matter were assembled into one black hole. Taking the most recent observational data, this would yield the entropy (calculated in the Appendix)

$$
S_{\max } \approx 1.8 \times 10^{121} .
$$

This may not yet be the maximal possible entropy. Our Universe exhibits currently an acceleration which could be caused by a cosmological constant $\Lambda$. If this is true, it will expand forever, and the entropy in the far future will be dominated by the entropy of the cosmological event horizon (the "GibbonsHawking entropy" [7]). The estimate, presented in the Appendix, yields

$$
S_{\mathrm{GH}}=\frac{3 \pi}{\Lambda l_{\mathrm{P}}^{2}} \approx 2.88 \times 10^{122},
$$

which is about one order of magnitude higher than (3).

Following the arguments in [6], the "probability" for our Universe can be estimated as

$$
\frac{\exp (S)}{\exp \left(S_{\max }\right)} \approx \frac{\exp \left(3.1 \times 10^{104}\right)}{\exp \left(2.9 \times 10^{122}\right)} \approx \exp \left(-2.9 \times 10^{122}\right) .
$$

Our Universe is thus very special indeed. It must have "started" near the Big Bang with an extremely low entropy; the Universe must have been very smooth in the past, with no white holes being present. Penrose has reformulated this observation in his Weyl-tensor hypothesis: the Weyl tensor is zero near the Big Bang (describing its smooth state), but diverges in a Big Crunch (provided the Universe will recollapse). Since the Weyl tensor describes in particular gravitational waves, this hypothesis entails that all gravitational waves must be retarded. This is analogous to the Sommerfeld condition in electrodynamics and the absence of advanced electromagnetic waves [1]. There, the 
electromagnetic arrow can be traced back to the thermodynamic arrow and the Second law by using the thermodynamic properties of absorbers, but this is not possible here because gravitational waves are too weak.

The Weyl-tensor hypothesis is not yet an explanation, but only a description of the low initial cosmic entropy. Penrose has recently reformulated his hypothesis in the framework of his "Conformal Cyclic Cosmology" (CCC) 8. A central role is attributed therein to a proposed information loss in black holes and the ensuing nonunitary evolution. His whole picture, however, remains classical as far as gravity is concerned. Here, I would like to adopt instead the point of view that the gravitational field is fundamentally of quantum nature. This is not a logical necessity, but one can put forward physical arguments in favour of quantum gravity as the more fundamental theory [9, 10]. Although there exist non-singular classical solutions, the singularity theorems of classical relativity suggest the abundance of singularities in the classical theory; a more general framework is therefore needed to exorcize them. Moreover, gravity acts universally, so it couples to all other fields of Nature, all of which are so far described by quantum theory. It could then at least be considered unnatural to leave the gravitational field classical; this would become especially awkward in the context of a unified theory of all interactions.

In the next section I shall briefly describe an approach to quantum gravity which is very conservative and which despite its limits should be able to provide insights into the origin of the arrow of time.

\section{Quantum Geometrodynamics}

A full quantum theory of gravity remains elusive 9 . Can one nevertheless say something reliable about quantum gravity without knowing the exact theory? In 11] I have made the point that this is indeed possible. The situation is analogous to the role of the quantum mechanical Schrödinger equation. Although this equation is not fundamental (it is non-relativistic, it is not fieldtheoretic), important insights can be drawn from it. For example, in the case of the hydrogen atom, one has to impose boundary conditions for the wave function at the origin $r \rightarrow 0$, that is, at the centre of the atom. This is certainly not a region where one would expect non-relativistic quantum mechanics to be exactly valid, but its consequences, in particular the resulting spectrum, are empirically correct to an excellent approximation.

Erwin Schrödinger has found his equation by "guessing" a wave equation from which the Hamilton-Jacobi equation of classical mechanics can be recovered in the limit of small wavelengths, analogously to the limit of geometric optics from wave optics. The same approach can be applied to general relativity. One can start from the Hamilton-Jacobi version of Einstein's equations and "guess" a wave equation from which they can be recovered in the classical limit. The only assumption that is required is the universal validity of 
quantum theory, that is, its linear structure. It is not yet needed for this step to impose a Hilbert-space structure. Such a structure is employed in quantum mechanics because of the probability interpretation for which one needs a scalar product and its conservation in time (unitarity). The status of this interpretation in quantum gravity remains open, see below.

The result of this approach is quantum geometrodynamics. Its central equation is the Wheeler-DeWitt equation, first discussed by Bryce DeWitt and John Wheeler in the 1960s. In a short notation, it is of the form

$$
\hat{H} \Psi=0,
$$

where $\hat{H}$ denotes the full Hamiltonian for both the gravitational field (here described by the three-metric) as well as all non-gravitational fields. For the detailed structure of this equation I refer, for example, to the classic paper by DeWitt [12, or the general review in 9]. Two properties are especially important for our purpose here. First, this equation does not contain any classical time parameter $t$. The reason is that spacetime as such has disappeared in the same way as particle trajectories have disappeared in quantum mechanics; here, only space (the three-geometry) remains. Second, inspection of $\hat{H}$ exhibits the local hyperbolic structure of the Hamiltonian, that is, the Wheeler-DeWitt equation possesses locally the structure of a Klein-Gordon equation. In the vicinity of Friedmann universes, this hyperbolic structure is not only locally present, but also globally. One can thus define a new time variable which exists only intrinsically and which can be constructed from the three-metric (and non-gravitational fields) itself. It is this absence of external time that could render the probability interpretation and the ensuing Hilbertspace structure obsolete in quantum gravity, for no conservation of probability may be needed 1

How, then, can one understand the emergence of an arrow of time from a fundamental equation which is itself timeless? I shall address this issue in the next section.

\section{Arrow of Time from Quantum Cosmology}

Quantum cosmology is the application of quantum theory to the Universe as a whole. In a first approximation, the Universe is homogeneous and isotropic. The three-metric is then fully characterized by the scale factor, $a$, of the Universe. Classically, the Universe evolves in time, $a(t)$; the same holds for the matter fields. In quantum cosmology, $t$ has disappeared and all available information is encoded in the wave function $\psi(a, \ldots)$, where the $\ldots$ denote

\footnotetext{
${ }^{1}$ The situation is different for an isolated quantum gravitational system such as a black hole; there, the semiclassical time of the rest of the Universe enters the description 13 .
} 
homegeneous matter degrees of freedom. For the simple two-dimensional configuration space consisting of the scale factor and a minimally coupled scalar field $\phi$, the Wheeler-DeWitt equation reads (with $c=1$ )

$$
\begin{aligned}
\hat{H} \Psi= & \left(\frac{2 \pi G \hbar^{2}}{3} \frac{\partial^{2}}{\partial \alpha^{2}}-\frac{\hbar^{2}}{2} \frac{\partial^{2}}{\partial \phi^{2}}\right. \\
& \left.+e^{6 \alpha}\left(V(\phi)+\frac{\Lambda}{8 \pi G}\right)-3 e^{4 \alpha} \frac{k}{8 \pi G}\right) \Psi(\alpha, \phi)=0,
\end{aligned}
$$

with cosmological constant $\Lambda$ and curvature index $k= \pm 1,0$. The variable $\alpha=\ln a$ has been introduced for convenience.

In order to discuss thermodynamical issues, additional degrees of freedom must be added. One option is to consider small inhomogeneities in the vicinity of homogeneity. This can be achieved, for example, through a multipole expansion on the three-sphere (assuming the Universe is closed) [14, 15. Schematically, the Wheeler-DeWitt equation (6) then assumes the form

$$
\hat{H} \Psi=\left(\frac{2 \pi G \hbar^{2}}{3} \frac{\partial^{2}}{\partial \alpha^{2}}+\sum_{i}[-\frac{\hbar^{2}}{2} \frac{\partial^{2}}{\partial x_{i}^{2}}+\underbrace{V_{i}\left(\alpha, x_{i}\right)}_{\rightarrow 0 \text { for } \alpha \rightarrow-\infty}]\right) \Psi=0,
$$

where the $\left\{x_{i}\right\}$ denote the scalar field as well as the inhomogeneous degrees of freedom; $V_{i}\left(\alpha, x_{i}\right)$ are the corresponding potentials. One recognizes immediately that this Wheeler-DeWitt equation is hyperbolic with respect to the intrinsic time $\alpha$. Initial conditions are thus most naturally formulated with respect to constant $\alpha$.

The important observation is now that the potential in (8) is asymmetric with respect to $\alpha$; if written out, it contains explicit factors of $\mathrm{e}^{6 \alpha}$, etc., and vanishes in the limit $\alpha \rightarrow-\infty$. In contrast to almost all the other fundamental equations in physics, it thereby distinguishes a direction in (intrinsic) time. One could thus envisage of solution to the Wheeler-DeWitt equation, which near the Big Bang would be an approximate product state between all degrees of freedom [1],

$$
\Psi \stackrel{\alpha \rightarrow-\infty}{\longrightarrow} \psi_{0}(\alpha) \prod_{i} \psi_{i}\left(x_{i}\right)
$$

Introducing the entropy of the Universe as an entanglement entropy, in which irrelevant, that is, unobservable or unobserved degrees of freedom (such as small gravitational waves described by some of the $x_{i}$ ) are integrated out, the state (9), which is a product state, would yield a vanishing entropy. For increasing $\alpha$, this solution would evolve into a superposition of $\alpha$ and the inhomogeneous modes (as well as between the inhomogeneous modes). Integrating out all or part of the $x_{i}$ would then yield a non-vanishing and increasing entropy with respect to $\alpha$. Increasing entanglement would then cause increasing decoherence for the relevant degrees of freedom [16, 15, 17]. Decoherence - the 
unavoidable emergence of classical properties through interaction with irrelevant degrees of freedom - is perhaps the most fundamental irreversible process and thus stands behind all arrows of time [1, 17. Because of the asymmetry of (8) with respect to $\alpha$, substituting $\alpha \rightarrow-\alpha$ would not yield a solution of the Wheeler-DeWitt equation. If a solution of the form (8) were the one describing our Universe, we could understand from it the irreversible appearance of our world. A full understanding of quantum gravity would perhaps single out a unique solution for the Wheeler-DeWitt equation, a possibility already envisioned by DeWitt [12].

There are indications that the above quantum state would evolve into a symmetric state where all perturbations are in an (at least approximate) de Sitter-invariant vacuum state. Such a vacuum state is a good candidate for the early Universe [18. The state for each perturbation mode would describe a superposition of inhomogeneous states, that is, a non-classical state. However, the mechanism of decoherence also comes into play here, generating a classical behaviour for the modes which may then serve as the seeds for the origin of structure in the Universe [19.

We have not yet discussed the connection between the intrinsic time $\alpha$ and the "observed time" $t$ which should be at our disposal at least in an appropriate semiclassical limit. This can be achieved through a Born-Oppenheimer type of approximation scheme, cf. [9] and the references therein. Some degrees of freedom such as the scale factor may serve as the semiclassical variables from which a semiclassical time $t$ can be defined in appropriate situations. This will be the time variable which controls the dynamics in this approximation and which enters an effective and approximate Schrödinger equation for the non-gravitational quantum variables. The arrow of time aligned along increasing scale factor $a$ thus trivially extends to the semiclassical time $t$ - as long as the semiclassical approximation is valid.

The above ideas may, with slight elaborations, also apply to the idea of the multiverse, that is, to a Universe with many approximately homogeneous sub-universes, cf. [5] and the references therein. Quantum entanglement is not limited to sub-horizon scales and may thus be effective also in the full multiverse. Decoherence should then distinguish the same arrow of time everywhere in the multiverse. Applying also here the idea that quantum fluctuations, after their effective classicality due to decoherence, become the seeds for galaxy formation, Linde and Vanchurin estimate in [5] the number of realizations of the emergent classical fluctuations in the gigantic multiverse. This number would also correspond to the number of branches of the universal wave function in the Everett interpretation when applied to our Hubble domain. After decoherence, each realization can serve as a classical initial condition for the subsequent evolution of the Universe. They find for the total number of distinguishable locally "Friedmann universes" the estimate

$$
\mathrm{e}^{S_{\text {pert }}} \lesssim \mathrm{e}^{\mathrm{e}^{3 N}},
$$


where $S_{\text {pert }}$ is the total entropy of the perturbations, see also [19, 20], and $N$ is the number of e-folds of slow-roll (post-eternal) inflation. In the simplest models of chaotic inflation, one thereby gets the incredibely high number [5]

$$
10^{10^{10^{7}}} \text {. }
$$

(A much lower number - two instead of three exponentials - is obtained in the case of a positive cosmological constant.) Adopting, in addition, the landscape picture of string theory, this estimate would correspond to the case of one vacuum. Taking all the vacua into account, the number will be even higher. The issue of the Wheeler-DeWitt equation on a configuration space mimicking the landscape picture and the question of a low-entropy initial condition was discussed, for example, in [21.

The idea of quantum cosmology is that the whole Universe at all scales is described by quantum theory. Thus, a priori, quantum effects are not restricted to the Planck scale. In the case of a classically recollapsing quantum universe, for example, one can predict the occurrence of quantum effects near the classical turning point [22, see also [1, 23] for a detailed explanation. Because the arrow of time in the above scenario is correlated with increasing scale factor $a$, the quantum universe would in this case consist of many branches in which the arrow of time always points in the direction of increasing $a$. These branches would be decohered components of the universal wave functions and would thus become independent of each other for most of their existence, but they would interfere destructively at the classical turning point in order to fulfill the boundary condition $\Psi \rightarrow 0$ for $a \rightarrow \infty$, which is necessary for a model in which the classical trajectories recollapse. Consequently, no classical observers would be able to survive a transition through the turning point, and time as well as the classical evolution would come to an end there.

This is an impressive example for a quantum effect at large scales. Other examples can be found in models that are of interest because they may describe a dynamical dark energy in our Universe. Examples are models which classically exhibit a big-rip or a big-brake singularity; in the first case, the Universe can become infinitely large in a finite time, while in the second case it comes to an abrupt halt in the future. In both cases, this corresponds to a singular region. One can now study solutions of the corresponding WheelerDeWitt equations and finds that the singularities will be avoided, cf. [23] and the references therein: the semiclassical approximation breaks down when approaching the region of the classical singularity, and for the big brake the wave function even becomes zero there. What are the consequences of this scenario for the arrow of time?

Since the semclassical time comes to an end, so does the arrow. The Universe enters a genuine quantum era which no classical observers (and others are not known) could survive. This is analogous to the above discussed turning point. The world then becomes truly timeless.

One might wonder what happens in the case of models which classically describe bouncing cosmologies [24]: the Universe would then undergo many, 
perhaps infinite, cycles of expansion and recollapse. What would happen with the entropy in these cases? If the entropy were indeed correlated with the scale factor, as the scenario discussed above suggests, the arrow of time would not continue through a turning point. The bouncing models would thus make no sense in quantum cosmology; one would only have branches of the wave function in which the arrow would point from small to large universe and where time would end when approaching a classical turning point.

We have restricted the discussion to quantum geometrodynamics. At least for scales above the Planck length, which includes the above discussed quantum scenarios for a big universe in the future, this should provide a reliable framework. Modifications are, however, expected when approaching the Planck regime, that is, the region of the Big Bang. Such modifications have been addressed in string theory and loop quantum gravity [9]. In the case of loop quantum cosmology, the Wheeler-DeWitt equation is replaced at the most fundamental level by a difference (instead of differential) equation. For a big universe, the differences to quantum geometrodynamics are negligible; this concerns, for example, the examples of the big rip and the big brake. Near the big-bang singularity, however, the situation is different. The emerging scenario is discussed at length in another contribution to this volume [25]. Also there, the author suggests "the possibility of deriving a beginning within a beginningless theory". Thus, although the approaches may be different, the common fundamental challenge is to understand the observed time and its arrow from a scenario of the world which is fundamentally timeless.

\section{Acknowledgements}

I thank Max Dörner and Tobias Guggenmoser for a careful reading of this manuscript.

\section{A Some Numerical Estimates}

Here we recapitulate the numerical estimates about the maximal possible entropy in the Universe, presented by Penrose in [6], in the light of recent cosmological data [26. Since our Universe is spatially flat to a high degree of accuracy, the mass of the matter (both visible and dark) in our present Hubble volume is given by

$$
M_{\mathrm{U}}=\frac{4 \pi \rho_{\mathrm{m}} c^{3}}{3 H_{0}^{3}},
$$

where $\rho_{\mathrm{m}}$ is the matter density, and $H_{0} \approx 70.5 \mathrm{~km} / \mathrm{s} \mathrm{Mpc} \approx 2.27 \times 10^{-18} \mathrm{~s}^{-1}$ is the Hubble constant. Introducing the critical density

$$
\rho_{\mathrm{c}}=\frac{3 H_{0}^{2}}{8 \pi G},
$$


we can use the density parameter $\Omega_{\mathrm{m}}=\rho_{\mathrm{m}} / \rho_{\mathrm{c}} \approx 0.274$ and write

$$
M_{\mathrm{U}}=\frac{c^{3} \Omega_{\mathrm{m}}}{2 G H_{0}} \text {. }
$$

In order to estimate the maximal entropy, we shall assume that the Universe up to the Hubble scale consists of one black hole with mass $M_{\mathrm{U}}$. Since our present Universe is dominated by dark energy, which for our purpose here can be approximated by a cosmological constant $\Lambda$, we have to take into account that the metric outside this hole is, in fact, the Schwarzschild-de Sitter metric, see, for example, 27 . Numerically we have $\Lambda \approx 1.25 \times 10^{-56} \mathrm{~cm}^{-2}$ and $\Omega_{\Lambda}=\Lambda c^{2} / 3 H_{0}^{2} \approx 0.726$ [26]. In the Schwarzschild-de Sitter metric, the black hole has a maximal mass given by

$$
M_{\mathrm{N}}=\frac{c^{3}}{3 \sqrt{3 \Omega_{\Lambda}} G H_{0}} \approx 4 \times 10^{55} \mathrm{~g},
$$

which corresponds to the case of the Nariai metric (therefore the index N). We thus have to check whether $M_{\mathrm{U}}$ is greater or smaller than $M_{\mathrm{N}}$; only in the latter case can the Universe accommodate one single black hole. A short calculation shows

$$
\frac{M_{\mathrm{U}}}{M_{\mathrm{N}}}=\frac{3 \sqrt{3 \Omega_{\Lambda}} \Omega_{\mathrm{m}}}{2} \approx 0.61,
$$

so all of $M_{\mathrm{U}}$ can indeed be assembled into one black hole.

We now assume that the maximal entropy is given by a Schwarzschild black hole with mass $M_{\mathrm{U}}$ (a non-vanishing angular momentum would give a smaller entropy). Thus,

$$
S_{\max }=\pi\left(\frac{R_{\mathrm{h}}}{l_{\mathrm{P}}}\right)^{2},
$$

where $R_{\mathrm{h}}$ denotes the radius of the black-hole event horizon (as opposed to the cosmological horizon $R_{\mathrm{c}}$ ). In the Schwarzschild-de Sitter metric we have

$$
R_{\mathrm{h}}=\frac{3 G M_{\mathrm{U}} \ell \xi}{c^{2}}\left(1-\sqrt{1-\frac{1}{\ell \xi^{3}}}\right)=\frac{\xi}{\sqrt{\Lambda}}\left(1-\sqrt{1-\frac{1}{\ell \xi^{3}}}\right),
$$

where $\ell^{-1}=M_{\mathrm{U}} / M_{\mathrm{N}}$ and $\xi=\cos \left(\frac{1}{3} \cos ^{-1}\left[\ell^{-1}\right]\right) \approx 0.95$. With the above numbers we have

$$
R_{\mathrm{h}} \approx 2.13 \frac{G M_{\mathrm{U}}}{c^{2}} \approx 0.38 \times 10^{28} \mathrm{~cm}
$$

and therefore

$$
S_{\max } \approx 1.8 \times 10^{121} .
$$

This is the number that should replace the estimate $10^{123}$ in [6] if one makes use of the data presented in [26. 
Expressed in grams, the mass (11) is $M_{\mathrm{U}} \approx 2.4 \times 10^{55} \mathrm{~g}$ and therefore corresponds to about $1.5 \times 10^{79}$ baryons. In the case of $10^{80}$ baryons, as used in $\left[8\right.$, one would find $\ell^{-1}>1$, that is, the corresponding mass would exceed the Nariai mass (14) and it would thus not be possible to assemble this mass into a single black hole.

In the case of a non-vanishing cosmological constant there occurs also the gravitational entropy $S_{\Lambda}$ associated with the cosmological event horizon $R_{\mathrm{c}}$ 7], where

$$
R_{\mathrm{c}}=\frac{3 G M_{\mathrm{U}} \ell \xi}{c^{2}}\left(1+\sqrt{1-\frac{1}{\ell \xi^{3}}}\right) \approx 1.29 \times 10^{28} \mathrm{~cm} .
$$

It reads

$$
S_{\Lambda}=\pi\left(\frac{R_{\mathrm{c}}}{l_{\mathrm{P}}}\right)^{2} \approx 1.99 \times 10^{122} .
$$

One should thus in principle consider the sum of $S_{\Lambda}$ and the entropy associated with all matter being trapped in a single black hole. However, the maximal entropy is reached for asymptotic times $t \rightarrow \infty$ when the matter content becomes irrelevant (because it will be diluted and no black hole with mass $M_{\mathrm{U}}$ will be formed); the radius of the event horizon then approaches

$$
R_{\mathrm{c}}=\sqrt{\frac{3}{\Lambda}} \approx 1.55 \times 10^{28} \mathrm{~cm} .
$$

The entropy associated with this event horizon then approaches the "GibbonsHawking" entropy [7]

$$
S_{\mathrm{GH}}=\frac{3 \pi}{\Lambda l_{\mathrm{P}}^{2}} \approx 2.88 \times 10^{122},
$$

which is about 16 times the black-hole maximal entropy (19). The numerical value in (23) is also presented in [4.

Taking the case of the Nariai mass (14), one would have the total entropy $S_{\mathrm{N}}+S_{\Lambda}=2 S_{\mathrm{GH}} / 3$, which would give further support to consider (23) as the maximal possible entropy of the observable Universe, as suggested by current observational data.

\section{References}

1. H. D. Zeh (2007): The physical basis of the direction of time, fifth edition (Springer, Berlin).

2. H. D. Zeh (2009): Open questions regarding the arrow of time. Contribution to this volume, see also arXiv:0908.3780 v2 [gr-qc].

3. A. De Simone, A. H. Guth, A. Linde, M. Noorbala, M. P. Salem, and A. Vilenkin (2008): Boltzmann brains and the scale-factor cutoff measure of the multiverse. arXiv:0808.3778v1 [hep-th]. 
4. C. A. Egan and C. H. Lineweaver (2009): A larger estimate of the entropy of the universe. arXiv:0909.3983v1 [astro-ph.CO].

5. A. Linde and V. Vanchurin (2009): How many universes are in the multiverse? arXiv:0910.1589v1 [hep-th].

6. R. Penrose (1981): Time-asymmetry and quantum gravity. In Quantum gravity, Vol. 2, edited by C. J. Isham, R. Penrose, and D. W. Sciama, pp. 242-272 (Clarendon Press, Oxford).

7. G. W. Gibbons and S. W. Hawking (1977): Cosmological event horizons, thermodynamics, and particle creation. Phys. Rev. D 15, 2738-2751.

8. R. Penrose (2009): Black holes, quantum theory and cosmology. J. Phys.: Conference Series 174, 012001.

9. C. Kiefer (2007): Quantum Gravity, second edition (Oxford University Press, Oxford)

10. M. Albers, C. Kiefer, and M. Reginatto (2008): Measurement analysis and quantum gravity. Phys. Rev. D 78, 064051.

11. C. Kiefer (2009): Quantum geometrodynamics: whence, whither? Gen. Relativ. Gravit. 41, 877-901; C. Kiefer (2009): Does time exist in quantum gravity? arXiv:0909.3767 1 [gr-qc].

12. B. S. DeWitt (1967): Quantum theory of Gravity. I. The canonical theory. Phys. Rev. 160, 1113-1148.

13. C. Kiefer, J. Marto, and P. V. Moniz (2009): Indefinite oscillators and black-hole evaporation. Ann. Phys. (Berlin) 18, 722-735.

14. J. J. Halliwell and S. W. Hawking (1985): Origin of structure in the Universe. Phys. Rev. D 31, 1777-1791.

15. C. Kiefer (1987): Continuous measurement of minisuperspace variables by higher multipoles. Class. Quantum Grav. 4, 1369-1382.

16. H. D. Zeh (1986): Emergence of classical time from a universal wave function. Phys. Lett. A 116, 9-12.

17. E. Joos, H. D. Zeh, C. Kiefer, D. Giulini, J. Kupsch, and I.-O. Stamatescu (2003): Decoherence and the Appearance of a Classical World in Quantum Theory, second edition (Springer, Berlin).

18. A. A. Starobinsky (1979): Spectrum of relict gravitational radiation and the early state of the universe. JETP Lett. 30, 682-685.

19. C. Kiefer, I. Lohmar, D. Polarski, and A. A. Starobinsky (2007): Pointer states for primordial fluctuations in inflationary cosmology. Class. Quantum Grav. 24, 1699-1718; C. Kiefer and D. Polarski (2009): Why do cosmological perturbations look classical to us? Adv. Sci. Lett. 2, 164-173, see also arXiv:0810.0087v2 [astro-ph].

20. C. Kiefer (2001): Entropy of gravitational waves and primordial fluctuations. In: Cosmology and particle physics, ed. by J. Garcia-Bellido, R. Durrer, and M. Shaposhnikov (American Institute of Physics, New York), pp. 499-504.

21. R. Holman and L. Mersini-Houghton (2006): Why the Universe started from a low entropy state. Phys. Rev. D 74, 123510 .

22. C. Kiefer and H. D. Zeh (1995): Arrow of time in a recollapsing quantum universe. Phys. Rev. D 51, 4145-4153.

23. C. Kiefer and B. Sandhöfer (2009): Quantum cosmology. In: Beyond the Big Bang, ed. by R. Vaas (Springer, Berlin), see also arXiv:0804.0672 2 [gr-qc].

24. M. Novello and S. E. P. Bergliaffa (2008): Bouncing cosmologies. Phys. Rep. 463, 127-213. 
25. M. Bojowald (2009): A momentous arrow of time. Contribution to this volume, see also arXiv:0910.3200v1 [gr-qc].

26. G. Hinshaw et al. (2009): Five-Year Wilkinson Microwave Anisotropy Probe (WMAP) Observations: Data Processing, Sky Maps, and Basic Results. Astrophys. J. Suppl. 180, 225-245.

27. K. H. Geyer (1980): Geometrie der Raum-Zeit der Maßbestimmung von Kottler, Weyl und Trefftz. Astronomische Nachrichten 301, 135-149. 